\title{
Reducing Computational Cost and Allowing Automatic Remeshing in FEM Models of Metal Forming Coupled With Polycrystal Plasticity
}

\author{
R.E. Logé ${ }^{1}$, M. Béringhier ${ }^{1}$, Y.B. Chastel ${ }^{1}$ and L. Delannay ${ }^{2}$ \\ 1 - Centre de Mise en Forme des Matériaux (CEMEF), UMR CNRS 7635, Ecole des Mines de Paris, B.P. 207, \\ 06904 Sophia Antipolis Cedex, France (Roland.Loge@ensmp.fr) \\ 2 - CESAME - MEMA, Université catholique de Louvain (UCL), bâtiment Euler, av. G. Lemaître 4, 1348 Louvain- \\ la-Neuve, Belgium
}

\begin{abstract}
The paper proposes an original use of the Lagrangian particles concept for finite element computation of microstructure evolution in metal forming. The method amounts to distributing incomplete representations of the microstructure among the integration points of the mesh while a complete microstructure is associated with each Lagrangian particle. This decreases the computation time and enables the transport of microstructural variables when remeshing. While the method is presented for any kind of discretized microstructure, it is applied here to the prediction of mechanical anisotropy induced by crystallographic texture. In this specific case, the numerical predictions are validated against experiment by considering compression of a textured aluminium alloy (AA7175). The model accuracy is assessed with respect to mechanical anisotropy but texture evolution is also considered.
\end{abstract}

Keywords: Lagrangian particles, microstructure, finite element, anisotropy, crystallographic texture, computation time, remeshing

PACS:

\section{INTRODUCTION}

When introducing advanced material behaviour models into finite element descriptions of forming processes, an important challenge is to account for the limitations in computing power and memory. Indeed, these models often rely on some discretized representation of the microstructure, using a homogenization technique to predict mechanical behaviour and microstructure evolution. This requires large computation times, usually in direct relation with the number of variables used to represent the microstructure. The total number of variables increases with the accuracy of the material description and with the complexity of the homogenization model.

A typical example is given by polycrystalline microstructures described by a crystallographic texture. For numerical simulations, the texture is often represented by a discrete set of orientations or 'representative grains'. Homogenization schemes based on crystal plasticity theory enable computing the mechanical anisotropy of the metallic material from the texture and a few complementary variables, e.g.
[1]. They account for strain-induced texture evolution, and hence predict the evolution of the mechanical anisotropy during the metal forming operation.

Various strategies can be adopted in order to predict the evolving mechanical anisotropy in the context of the finite element modelling of a forming process. A first scheme assigns to each integration point a representative volume of the polycrystalline material, where a collection of $N$ crystallographic orientations is used to represent the texture [2]. Even when starting with a homogeneous initial texture, i.e. with the same $N$ orientations at each integration point, distinct evolutions of the mechanical behaviour can be predicted from one integration point to another, as the texture evolves according to the local deformation path. A second type of approach allows avoiding the systematic use of the homogenization technique at each strain increment. A yield surface is constructed from the polycrystalline homogenization scheme [3], and updated only when significant deformation has taken place. However, the delayed yield surface reconstruction is itself computationally heavy [4]. Strategies have therefore been developed to restrict the definition of the yield surface to a narrow region in the 
stress space [5]. A way to efficiently feed textures into finite element simulations of forming processes has been proposed by Raabe and al. [6]. The objective was to reduce redundant information. Using the "texture component method", the authors were able to convert the measured initial texture into a reduced set of crystallographic orientations, which were then mapped onto the integration points of the global finite element mesh. Although there seemed to be difficulties related to the assignment of a random texture component, the approach provided valuable results when applied to the cup drawing process.

The method presented in this work was already tested in several preliminary studies [7-9]. The texture information is distributed throughout the finite element mesh, assigning to each integration point only a subset of the microstructure description. The initial texture is discretized into a finite set of orientations (see e.g. [10]), which are then mapped randomly onto the integration points. The texture associated to a single integration point is not representative (it is an 'incomplete' or 'partial texture'), but texture heterogeneity is cancelled out over several integration points so that the collective mechanical behaviour is adequate. The following 'academic' validations of the distribution procedure were investigated: uniaxial compression of a cube without friction [7], uniaxial compression of a cylinder with friction [8], and deepdrawing of a cup [9]. A strong decrease of computation time was obtained, as well as a valid prediction of mechanical anisotropy and texture evolution as long as a minimum number of orientations was mapped to each integration point. A similar technique was applied to the prediction of forming limit diagrams [11].

However, no solution was yet proposed for processes which require remeshing. It is precisely in those cases, where large deformations are involved, that the evolution of microstructure is significant, and must be computed to describe the corresponding evolution of anisotropy. Hence, crystal-plasticitybased finite element simulations have here a definitive advantage over other methods deriving mechanical anisotropy from the macroscopic yield locus. The concept of Lagrangian particles combined with the use of reduced orientation samplings [7-9] allows the transport of microstructural information when (automatic) remeshing occurs. The term 'Lagrangian' means that the particle, initially placed at some location in the mesh, will move according to the local deformation field. Lagrangian particles have historically been introduced in the context of fluid computations and Eulerian frameworks [12]. The method has been adapted more recently within an Arbitrary Lagrangian Eulerian framework formulation [13].
In the present work, the particles are used within a Lagrangian finite element formulation, i.e. their displacement is the same as that of the mesh. For this reason, Lagrangian particles allow a spatial localization of the microstructural information that is independent of the finite element mesh and that can therefore be used to transport the microstructural variables when remeshing occurs.

In summary, the purpose of the present paper is to discuss the use of incomplete microstructure representations distributed throughout a FE mesh. Compared to earlier studies by the same authors [7-9], the method is here presented in wider framework applicable to a large class of microstructures. Lagrangian particles are introduced, and results are validated against experimental data. The general method is described in Chapter 2. The specific case of polycrystalline materials in which microstructure is principally described by a set of crystallographic orientations and their volume fractions is treated in Chapter 3. Chapter 4 finally illustrates the concept within an example, where results are analyzed and discussed.

\section{LAGRANGIAN PARTICLES AND MICROSTRUCTURE EVOLUTION}

For generality purposes, the method is presented without relying on the characteristics of a specific microstructure. It is assumed that a discrete representation of the microstructure is available and that the coupling between mechanical and microstructural evolution can be computed within the framework of the finite element technique.

\section{Microstructure Representation Within The Finite Element Mesh}

Some particles, defined by their spatial positions, are initially distributed over the volume, as illustrated in Figure 1. These particles are Lagrangian in the sense that when the volume is deformed, they follow the material yield, and hence the mesh in a Lagrangian finite element formulation (see Figure 1). We can therefore associate microstructure information to the particles, this information being correctly localized throughout the deformation.

To properly define the coupling between mechanical and microstructural evolution, we need to transfer microstructure information from the particles to the integration points. For this purpose, we define a cell associated to each particle. Each cell is defined as the subset of integration points of the mesh which are closer to this particle than to any other particle, using the usual Euclidean distance (see Figure 1 where two 
cells are sketched). A representative sampling of the microstructure is attached to each particle, and is partitioned amongst the different integration points contained in the cell. The sampled microstructures considered at the integration points are incomplete while their union is statistically representative within a cell. The evolution of the representative microstructure is obtained at the scale of the cells. This allows the particles to indeed stand for a spatial localization of microstructural information.

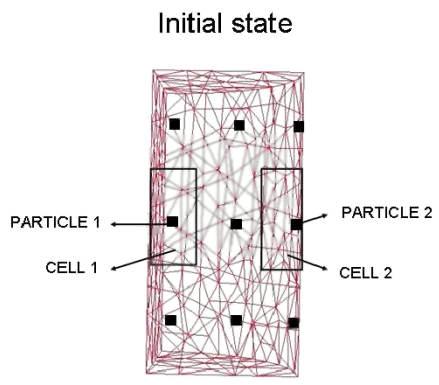

Deformed state

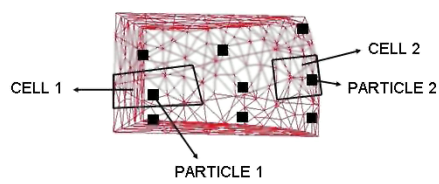

FIGURE 1. Initial and final state of $1 / 8^{\text {th }}$ of a cylinder under uniaxial compression. Lagrangian particles are represented by black squares. The evolution of two cells is highlighted.

Let us consider a microstructure consisting of $N$ phases. Physical properties of each phase are characterized by $k$ scalar, vectorial or tensorial variables. Geometrical properties are quantified through shape functions depending on $d$ characteristic lengths and $a$ characteristic angles. A topological description can also include correlation functions, giving the probability for neighbouring points in space to display different physical or geometrical properties, and modelled by $\Gamma$ variables. The description of the microstructure then requires $N_{V}=N(k+d+a)+\Gamma$ variables. Let us however assume that no topological description of the microstructure is available, except the volume fraction of each phase (zero-order correlation function), with therefore $\Gamma=N$. Higher order correlation functions will require treatments that can be adapted from the considerations below.

The distribution occurs at the phase level : each phase is associated to a single integration point that is selected randomly within the cell. However, the coupling between mechanical and microstructural evolution requires that the sum of the volume fractions of the phases attributed to an integration point be proportional to the volume associated to the integration point (see Figure 2.b). Let us discuss this further.
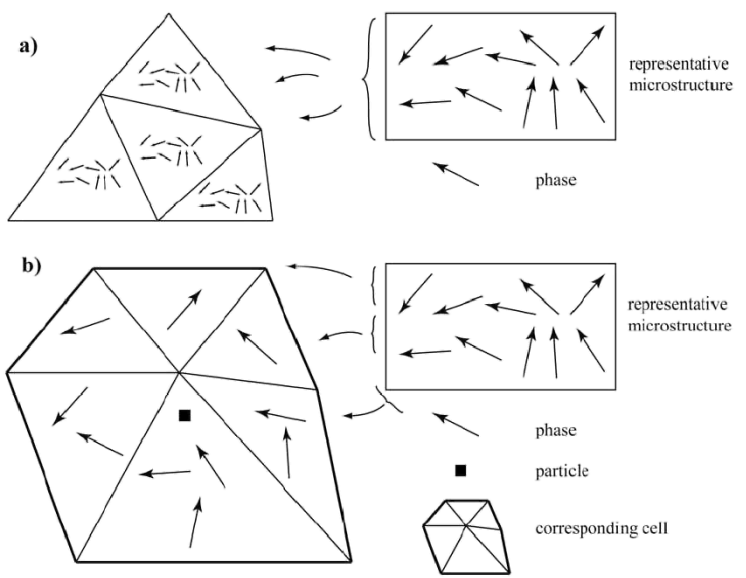

FIGURE 2. Illustrations (assuming one integration point per finite element) of (a) a complete microstructure being associated to each integration point, and (b) the distribution of the phases into a cell.

Assume that the phases all have the same volume fraction, normalized to 1 . Then consider that there are $C$ cells, and that the discretized microstructure is distributed $M_{c e l}$ times into the cell labelled $c e l$, and thus $M_{t o t}=\sum_{c e l=1, C} M_{c e l}$ times throughout the mesh. In order to reduce computation time in comparison to the usual method (complete microstructure at each integration point), we consider that the numbers $C$ and $M_{c e l}$ are small enough so that $M_{t o t}$ is smaller than the total number of integration points in the mesh. Hence $N_{c e l}=M_{c e l} N$ phases (resp. $N_{\text {tot }}=M_{\text {tot }} N$ phases) are distributed among the integration points in the cell cel (resp. in the mesh). We note $N_{i}$ the number of phases distributed at the integration point $i, V_{t o t}$ the volume of the global mesh, $V_{c e l}$ the volume of the cell $\mathrm{cel}$, and $V_{i}$ the volume associated to the integration point $i$.

Since the microstructure information associated to each integration point is incomplete, spatial zones where a representative microstructure can be found contain several integration points, among which we want the volume fractions of the phases to be equal. Then, by transitivity, three equations must be satisfied in order to respect volume fractions between :

- the integration points over the whole mesh :

$$
\forall i, N_{i} \cong N_{\text {tot }} \frac{V_{i}}{V_{\text {tot }}}
$$

- the cells over the whole mesh :

$$
\forall \mathrm{cel}, M_{c e l} \cong M_{\text {tot }} \frac{V_{c e l}}{V_{\text {tot }}}
$$

- the integration points in each cell cel: 


$$
\forall i \in c e l, N_{i} \cong N_{c e l} \frac{V_{i}}{V_{c e l}}
$$

In these equations, the lefthand term is an integer, while the righthand term is a real number. Depending on the "arithmetics" of the quantities $\left(\frac{V_{i}}{V_{t o t}}\right)_{i}$, $\left(\frac{V_{c e l}}{V_{t o t}}\right)_{c e l}$, the number $M_{t o t}$ must be large enough for the equations (1) and (2) to hold, and depending on the "arithmetics" of the terms $\left(\frac{V_{i}}{V_{c e l}}\right)_{i \in c e l}$, the number $M_{c e l}$ must be large enough for the equation (3) to hold. If we have only one particle $(C=1)$, equations (1) and (3) are equivalent, while equation (2) is trivial, meaning that we are left with only one equation. With more than one particle, the error in (1) is a priori larger, since it cumulates the errors in (2) and (3), equation (1) being the product of equations (2) and (3). While it refines spatial localization, increasing the number of particles thus tends to increase the number $M_{\text {tot }}$ required for a good representation of phase volume fractions.

In the case where volume fractions are different among the phases, the same equations apply, except that $N_{\text {tot }}, N_{c e l}$ and $N_{i}$ will be the sums of the volume fractions of the phases instead of the number of phases. These sums being real numbers, they depend on the choice of the particular phases associated with each integration point.

\section{Microstructure Evolution}

Once the phases are distributed, the partial microstructure associated with an integration point evolves depending on the local mechanical fields values. In a cell, the representative microstructure hence evolves depending on the mechanical conditions at its integration points. The cell must therefore represent an area where the mechanical fields are sufficiently homogeneous. This means that the choice of the particles locations in the mesh is important.

Taking into account this last consideration requires in general to increase the number $C$ of particles. On the other hand, the criteria (1), (2) and (3) tend to increase the required number $M_{\text {tot }}$ of distributed representative microstructures. However, both constraints lead to an overall number $N_{\text {tot }}$ of distributed phases which remains smaller than that of the classical approach where the complete microstructure is associated to each integration point. Accordingly, a significant decrease in computation time is observed, while maintaining an accurate mechanical response.

\section{Remeshing Management}

When the finite element mesh begins to degenerate, remeshing is needed, and state variables have to be transferred from the old mesh to the new one. Microstructure variables generally cannot be transported using classical spatial interpolation. For example, the conservation of the volume fractions of the phases during remeshing is problematic since there is neither bijection nor simple inclusion relation between the set of volumes associated with the integration points of the old mesh and the corresponding set for the new one.

The proposed approach is based on the spatial localization of the particles (see [14] for more details). As the positions of the particles are known and fixed throughout the remeshing process, the procedure goes as follows when remeshing occurs :

- phases and their linked microstructural variables are gathered and attached to the particle they are associated with,

- cells are redefined from the new mesh integration points,

- for each particle, phases and their linked microstructural variables are randomly redistributed among the integration points of the new cell, in such a way that equation (3) is satisfied.

The localization of the microstructure information attached to a particle is conserved through the remeshing process. However, the phases being distributed randomly in the cell, their spatial position does change, which again calls for cells with relatively homogeneous velocity gradients.

\section{CASE OF POLYCRYSTALLINE MICROSTRUCTURES}

When microstructure is determined solely by its crystallographic texture, it is discretized into a finite number of crystallographic orientations. A polycrystalline model can be used to determine the mechanical response of the polycrystal and to compute the texture evolution. Let us consider $N$ crystallographic orientations representing the material microstructure. We can assimilate them to $N$ different phases since they have different mechanical responses. Depending on the choice of the polycrystalline model, each of these orientations can be characterized by :

- $k$ variables describing physical properties. Typically 3 Euler angles for the crystallographic orientation, the critical resolved shear stresses associated to the slip systems, and possibly crystalline stress values if the constitutive behaviour depends on both plasticity and elasticity. 
- $d+a$ variables describing morphology, typically

$3+3$ variables describing a representative ellipsoid.

- one volume fraction.

In terms of topological description, two-points correlation functions can be used for example with a 2sites self-consistent polycrystalline model as described by Lebensohn and Canova [15].

The hypothesis of equal volume fractions simplifies crystallographic texture discretization [16], and is classical when using polycrystalline plasticity theory. With this hypothesis, previous considerations on the microstructure representation apply. For practical purpose, we here specify how the corresponding criteria can be satisfied. Given a total number $M_{\text {tot }}$ of distributed representative textures, $M_{c e l}$ representative textures are first distributed at each cell cel, with :

$$
M_{c e l}=E\left(M_{t o t} \frac{V_{c e l}}{V_{t o t}}\right)
$$

where $E()$ is the floor function. There are

$$
M_{r}=M_{t o t}-\sum_{c e l} E\left(M_{t o t} \frac{V_{c e l}}{V_{t o t}}\right)
$$

remaining textures to be distributed. We attribute one of them to the $M_{r}$ cells with the largest values of

$$
\alpha_{c e l}=\left(M_{t o t} \frac{V_{c e l}}{V_{t o t}}-M_{c e l}\right) / M_{c e l} .
$$

$M_{\text {tot }}$ is set at a value as small as possible but which is large enough to keep the resulting error in equation (2) small. The same method is then applied to the distribution of orientations among the integration points in a cell. If the resulting error in equation (3) is small enough, then the error in equation (1) is also small. If it is too large, $M_{c e l}$ must be increased and the overall distribution of textures among the cells must then be reconsidered for relation (2) to hold.

\section{ILLUSTRATIVE EXAMPLE}

The approach is illustrated by considering a compression test [17-18]. A cylindrical aluminium alloy specimen is forged, the compression axis being perpendicular to the cylinder axis (see Figure 3).

\section{Finite Element Model}

The simulation is performed with the Forge $3 \AA$ finite element code. This code uses a mixed velocitypressure formulation and the $\mathrm{P} 1+/ \mathrm{P} 1$ tetrahedral minielement. The polycrystalline model considered is an elasto-viscoplastic Taylor model, whose crystal plasticity algorithm can be found in [1]. For this specific application, the microstructure variables for each representative grain are : (a) 3 Euler angles describing the crystallographic orientation, (b) critical resolved shear stresses of potentially active slip systems for the considered material, and (c) the crystal stress tensors, with initial values assumed to be negligible (no initial residual stress).

The considered material is an extruded aluminium alloy AA7175, with initial axisymmetric pronounced texture. The texture has been discretized into a sampling of $N=1000$ orientations, with the same volume fraction. Dislocation slip rates in the slip planes are calculated based on a viscoplastic exponential law :

$$
\dot{\gamma}=\dot{\gamma}_{0}\left(\frac{\tau}{\tau_{c}}\right)^{1 / m} \operatorname{sign}(\tau)
$$

where $\dot{\gamma}$ is the shear strain rate, $\tau_{c}$ the critical resolved shear stress, $\tau$ the resolved shear stress, $\dot{\gamma}_{0}$ the characteristic strain rate and $m$ the strain rate sensitivity at the temperature of interest.

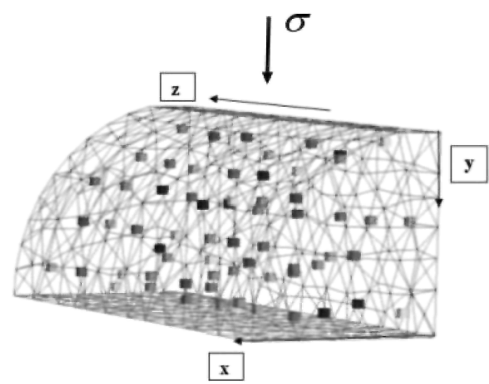

FIGURE3. Initial mesh of $1 / 8^{\text {th }}$ of a cylinder, with positioning of 63 particles.

The compression is done along the $y$-axis, as seen in Figure 3, with a final engineering strain of $60 \%$. The total number of remeshing operations during the simulation is 7. Two kinds of constitutive behaviour are tested: a viscoplastic isotropic behaviour and the elastic-viscoplastic Taylor model mentioned above. In both cases, the stress-strain curve of the material reaches the same level of stress at a given strain rate. With the polycrystalline model, we consider two numbers of particles, either 1 or 63 . The sampled texture is distributed 63 times in the mesh. In the case with 63 particles, particles are positioned such that all the corresponding cells have the same volume (Figure 3). One initial texture is considered at each particle, i.e. the texture is initially homogeneous in the specimen.

\section{Results And Discussion}

The mechanical anisotropy prediction is studied by comparison between experimental and numerical profiles according to three sections (x,y); (y,z) and $(\mathrm{x}, \mathrm{z})$. The profile obtained in the $(\mathrm{x}, \mathrm{z})$ section is 
represented in Figure 4. The numbers of particles considered in the model do not lead to noticeable differences in terms of flow anisotropy. Error analysis shows that the maximal relative error is $6.7 \%$ in the case of the polycrystalline rheology, and $10.86 \%$ in the case of the isotropic rheology. Relative errors are calculated on the $(x, y, z)$ positions of the external surface of the compressed specimen. The average of these relative errors is $3.3 \%$ in the case of the polycrystalline rheology and $7.84 \%$ in the case of the isotropic rheology. Hence, using the polycrystalline model allows to better predict mechanical anisotropy.

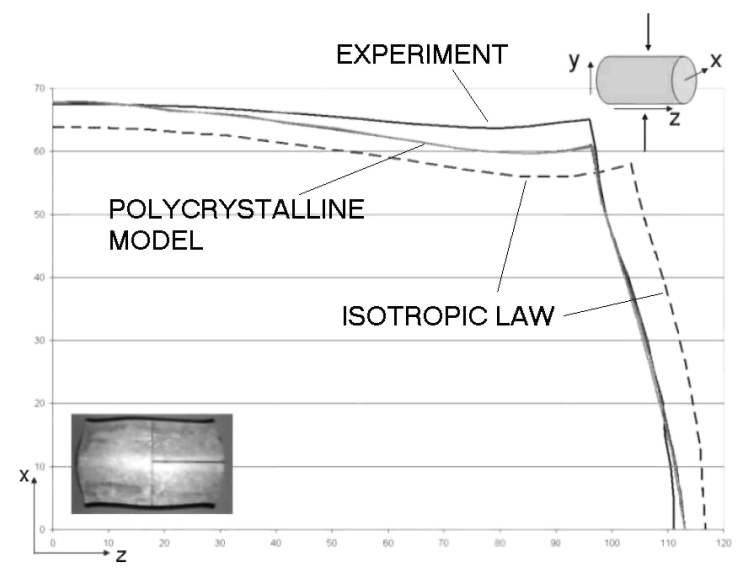

FIGURE 4. Profile of the $(x, z)$ section of the deformed cylindrical sample.

Texture evolution prediction was also analyzed (see [17] or [18] for more details). For both simulations and experiment, the crystallographic texture was measured in two areas : area 1 and area 2 . Area 1 , in the center of the sample, is more deformed than area 2 , on the side of the volume.

In the highly deformed area (area 1), all numerical pole figures were close to the experimental pole figures. In the smaller deformation area (area 2), the simulation with only 1 particle gave the same results as for area 1, due to the mixture of orientations at the scale of the global mesh during remeshing. In other words, with one particle, the orientations are redistributed over the entire volume at each remeshing event. When they fall in highly deformed areas, they evolve towards the texture of area 1, and when they fall in low deformation areas, they do not evolve much. Hence, after several remeshing operations, texture is always similar to that of area 1. This explains (a) the homogeneous texture in the mesh, and (b) the lower pole intensities in area 1 when using 1 particle, compared to that obtained with 63 particles.

The 63 particles case, where the orientations remain spatially localized at remeshing events shows the importance to consider a sufficient number of particles in order to capture microstructural heterogeneities in the mesh. With only one particle, the mechanical anisotropy is correctly predicted at the macroscopic scale of the specimen shape, after a strain of $60 \%$ (in Figure 4, the polycrystalline model results are similar with 1 and 63 particles). However it is expected that further deformation would finally lead to discrepancies, as local textures are no longer valid.

\section{ACKNOWLEDGMENTS}

The authors would like to thank Richard Ducloux and Patrice Lasne from Transvalor Company for stimulating ideas and fruitful discussions. L. Delannay is mandated by the National Found for Scientific Research (Belgium). M. Beringhier acknowledges support from the French "Simulforge" consortium.

\section{REFERENCES}

1. Delannay L, Jacques P.J.and Kalindidi S R 2006 Int. J. Plasticity 22 1879-98

2. Dawson P R, MacEwen S R and Wu P D 2003 International Material Reviews 48(2) 86-122

3. Van Houtte P, Mols K, Van Bael A and Aernoudt E 1989 Textures and Microstructures 11 23-39

4. Van Bael A, Winters J and Van Houtte P 1996 Textures of Materials, Proc. of the 11th Int. Conf. on Textures of Materials 1 eds. Liang Z, Zuo L, Chu Y, 356-61.

5. Habraken A M and Duchêne L 2004 Int. J. Plasticity 20 1525-60

6. Raabe D, Wang Y and Roters F 2005 Computationnal Materials Science 34 221-34

7. Béringhier M, Delannay L, Chastel $\mathrm{Y}$ and Logé R E 2004 Proc of the 7th ESAFORM Conf. eds. S. Stören, Trondheim, Norway, 167-74

8. Logé R E, Béringhier M, Chastel $\mathrm{Y}$ and Delannay L 2004 Proc. of NUMIFORM 2004 eds. S. Gosh and al., Columbus, Ohio, USA, 1754-59

9. Delannay L, Béringhier $\mathrm{M}$, Chastel $\mathrm{Y}$ and Logé R.E 2005 Materials Science Forum 495-497 1639-44

10. Toth L S and Van Houtte P 1992 Textures and Microstructures 19 229-44

11. Viatkina E M, Brekelmans W A M and Geers M G D 2005 J. Mat. Proc. Techn. 168(2) 211-18

12. Harlow F H 1964 Methods in Computationnal Physics, Fundamental Methods in Hydrodynamics 3, F.S. Adler A., Rotenberg M., Ed., Academic Press, New-York

13. Traoré K 2001 Thèse de Doctorat, Ecole Nationale Supérieure des Mines de Paris, CEMEF

14. Béringhier M, Logé R, Delannay L and Chastel Y 2006 Computer Methods in Materials Science 6 133-139

15. Lebensohn R A and Canova G R 1997 Acta Materiala 45(9) 3687-94

16. Melchior M A and Delannay L 2006 Computational Materials Science 37 557-64

17. Béringhier M 2006 Thèse de Doctorat, Ecole Nationale Supérieure des Mines de Paris, CEMEF

18. Béringhier M, Delannay L, Chastel Y and Logé R 2007 Modelling Simul. Mater. Sci. Eng. 15 191-204. 